\title{
Achieving the Recommended Levels of Physical Activity and Sleep among American Adolescent Athletes.
}

Gregory Knell ( $\sim$ Gregory.Knell@uth.tmc.edu )

University of Texas Health Science Center at Houston (UTHealth) https://orcid.org/0000-0002-5067$544 \mathrm{X}$

Timothy J. Walker

University of Texas Health Science Center at Houston

Ashleigh M. Johnson

Seattle Children's Hospital

Scott O. Burkhart

Andrews Institute for Orthopaedics and Sports Medicine

John D. Polousky

Children's Health Andrews Insitute for Orthpaedics and Sports Medicine

\section{Research article}

Keywords: Adolescent < Age Groups, Sports and Recreation, Muscle Strength, Leisure Activities, Exercise, Behavioral Risk Factor Surveillance System

Posted Date: July 21st, 2020

DOI: https://doi.org/10.21203/rs.3.rs-43598/v1

License: (c) (1) This work is licensed under a Creative Commons Attribution 4.0 International License. Read Full License 


\section{Abstract}

Background. Few adolescents are getting sufficient levels of physical activity and sleep despite the well understood health benefits of achieving sufficient levels of both, concurrently. This may be related to the limited understanding on the utility of programs to concurrently improve physical activity and sleep in adolescent populations. This study aimed to determine whether sports participation was associated with achieving the recommended levels of sleep and physical activity among a representative sample of American adolescents.

Methods. Participants included a representative sample $(n=58,008)$ of American adolescents who selfreported sport-participation (0, 1, 2, 3 or more teams yearly), and recommended levels of physical activity (at least 60-minutes per day of moderate or vigorous intensity physical activity [MVPA], and at least 3days per week of muscle-strengthening activities [MSA]) and sleep ( $\leq 12-13$ years: 9 to $10+$ hours; $14-17$ years: $8-10+$ hours; $\geq 18$ years: $7-9$ hours). Multivariable logistic regressions were used to estimate the relative odds of meeting the physical activity (MVPA and MSA) and sleep recommendations (independently and in combinations) in sex stratum among those participating in sports versus nonparticipation.

Results. Overall, $49.2 \%$ (95\% Cl: $47.1 \%-51.4 \%$ ) of males and $38.6 \%(95 \% \mathrm{Cl}: 37.3 \%-40.0 \%)$ of females report not participating in any sport. Sport-participation differed by sex, age, ethnicity (Hispanic), and weight status. Those who play 1-sport team had higher odds (females: $\mathrm{OR}=1.95$ [95\% $\mathrm{Cl}=1.59-2.40]$; males: $\mathrm{OR}=2.20$ [1.91-2.52] of concurrently achieving recommendations for sleep and physical activity (MVPA and MSA) compared to those playing no sports teams, after adjustment. Measures of effect were larger for more sports teams played, indicating a possible dose-response relation.

Conclusions. Among this representative sample of American adolescents, sports participation was consistently associated with a healthier physical activity and sleep profile. Practitioners should consider encouraging sports participation to adolescents when possible, particularly among females, racial/ethnic minorities, and those with concerns about getting adequate sleep and/or physical activity.

\section{Introduction}

Physical activity and sleep are integral components of healthy development during childhood and adolescence.(1) Aside from sharing many of the same health-related effects, physical activity and sleep are related behaviors. Children who are more physically active tend to sleep better,(2) and those who sleep better tend to be more active.(3) Unfortunately, recent data suggest few children/adolescents are getting sufficient levels of physical activity and sleep. Roman-Viñas et al.(4) found that $44 \%$ of children/adolescents across various sites meet physical activity recommendations ( $\geq 60$ minutes/day of moderate intensity aerobic activity) and only $42 \%$ meet sleep duration recommendations (9-11 hours/night). When considered together, only $18 \%$ of children/adolescents spend the optimal amount of time physically active and sleeping. Among American participants, only $10 \%$ met recommendations for both physical activity and sleep.(5) 
Numerous studies have examined the interrelation between sleep and physical activity among adolescents.(6-8) However, few studies have examined programs to concurrently improve physical activity and sleep. Notably, a school-based intervention improved physical activity levels and sleep quality over time for students in the intervention group but not in the control group.(9) In addition, research among adolescent athletes suggest they experience better sleep compared to non-athletes.(10) Despite these promising results, most studies examining physical activity programs among adolescents have not included sleep as an outcome.(11) Given the potential role of sports among adolescents, more research is necessary to better understand the relation between sport participation, physical activity, and sleep among this group.

The purpose of this analysis is to determine whether participation in high school sports is associated with concurrently and independently achieving recommended levels of sleep and physical activity among a nationally representative sample of American adolescents. We hypothesize that adolescents who participate in sports will be significantly more likely to achieve the recommended levels of physical activity and sleep, both independently and concurrently.

\section{Methods}

Data for this analysis are from multiple waves $(2011,2013,2015$, and 2017) of the Youth Behavioral Risk Factor Surveillance System (YRBS). YRBS is a serial cross-sectional, school-based national survey of health-risk behaviors among American adolescents.(12) Data are collected bi-annually by the Centers for Disease Control and Prevention using trained data collectors to administer a self-reported questionnaire to students during regular school hours. Participation is voluntary and requires parental consent. All data are anonymous and are freely and publicly available. For this analysis, the unweighted number of participants in each year of data collection were as follows: $2011(n=15,425), 2013(n=13,583), 2015$ ( $n$ $=15,624), 2017(n=14,765)$. Participants were in grades $9-12$ and are a representative sample of US high-school age students. More details on YRBS methodology appear elsewhere.(12)

All variables used in this analysis were participant-reported. The primary exposure of interest was sport participation within the past 12-months and included any sport team run by school or community groups. The primary outcomes were meeting the recommended time for sleep and physical activity, which were examined independently and concurrently. For sleep, participants reported the number of hours they sleep on an average school night based on an ordered categorical response options in one-hour increments from 4 or less hours to 10 or more hours. Using this, the prevalence of meeting the age specific sleep recommendations was defined as 9 to 10 or more hours for those aged 12 and under to 13 years, 8 to 10 or more hours for those aged 14 to 17 years, and 7-9 hours for those aged 18 years and over, consistent with the National Sleep Foundation recommendations.(13) For physical activity, participants reported the number of days, over the past 7-days, they were physically active at least a moderate intensity level (e.g., "increased your heart rate and made you breathe hard some of the time") for at least 60 minutes per day. Those who reported 7-days were classified as meeting the recommendation for moderate- to vigorousintensity aerobic physical activity. Participants additionally reported the number of days during the past 7- 
days that they did "exercises to strengthen or tone [their] muscles, such as push-ups, sit-ups, or weight lifting?" Those who reported at least 3-days were classified as meeting the recommendation for muscle strengthening physical activity. Potential covariates included in the analysis were demographics (e.g., sex, age, race/ethnicity [non-Hispanic white, non-Hispanic black, Hispanic/Latino, other]), weight status (e.g., underweight [ $<5$ th percentile], healthy weight [5th to $<85$ th percentile], overweight [85th to $<95$ th percentile], obese [ $>=95$ th percentile]), screen use (television, computer/video game), and comorbidities (e.g., asthma diagnosis, depression symptoms).

\section{Statistical analysis.}

To minimize bias associated with missing data, multiple imputation with chained equations (MICE) was used to impute missing values.(14) Categorical variables (e.g., sex, race/ethnicity) were imputed using multinomial logistic regression, ordinal variables (e.g., sport participation, grade, age, days of physical activity, sleep time, TV time, and video game/computer time) were imputed using predictive mean matching, dichotomous variables (asthma diagnosis, depression symptoms) were imputed using logistic regression, and continuous estimates (BMI percentile) were imputed using linear regressions. The survey sampling weight was included as a predictor for missing values and imputations were conducted separately by survey year. Data were imputed using 10, 15, 20, and 25 iterations, to determine if the larger number of iterations stabilized the estimates. It was found that 10 iterations were sufficient, and were therefore used in all further analyses. YRBS survey weights were used in all analyses.

The proportion and $95 \%$ confidence intervals of sport participation were estimated in sex strata by potential covariates (i.e., age, race/ethnicity, weight status, television/computer screen-use, asthma diagnosis, and the presence of symptoms of depression). The relative odds of meeting the physical activity and sleep recommendations (independently and in combinations) were calculated using logistic regressions in sex stratum and were adjusted by the potential covariates. STATA 15.1 (StataCorp. 2017. Stata Statistical Software: Release 15. College Station, TX: StataCorp LLC) was used for all analyses.

\section{Results}

The prevalence estimates with $95 \%$ confidence intervals $(\mathrm{Cl})$ of students who participated in sports across all years of observation are presented in Table 1 (female) and Table 2 (male). Overall, 49.2\% (95\% Cl: $47.1 \%-51.4 \%)$ of females and $38.6 \%$ (95\% Cl: $37.3 \%-40.0 \%)$ of males report not participating in any school or community-based sport. These sex differences (males had a greater levels of participation) were consistent across characteristics of interest. The oldest aged participants (18 years and older) had the highest levels of non-participation (females: $58.3 \%$ [95\% Cl: $55.4 \%-61.3 \%]$; males: $43.5 \%$ [38.8\%-44.2\%]) among all the age groups, as did Hispanic/Latino students (females: $55.6 \%$ [95\% Cl: $55.4 \%-61.3 \%$ ]; males: $41.8 \%$ [39.5\%-44.0\%]) among the other racial/ethnic groups. Those students who were classified as healthy weight had the lowest prevalence of non-participation compared to the other weight statuses, and among females, those classified as obese had the highest prevalence of nonparticipation $(63.5 \%[95 \% \mathrm{Cl}=60.7 \%-66.4 \%])$. 
Table 1

Weighted prevalence of sports-participation among female high school students residing in the United States by relevant characteristics, National Youth Risk Behavior Survey, 2011-2017

\section{Characteristic}

Total Female sport participation, \% (95\% Cl)

$\begin{array}{lll}0 \text { teams } & 1 \text { team teams } & \begin{array}{l}3 \text { or more } \\ \text { teams }\end{array}\end{array}$

\section{Overall}

28,784

$49.2(47.1-$ $51.4)$

$25.8(24.8-$

$15.2(14.2-$

$9.8(8.7-$ 26.8)

16.2)

11.0)

Age, years

\begin{tabular}{|c|c|c|c|c|c|}
\hline$<=13$ & 65 & $\begin{array}{l}49.7(35.6- \\
63.8)\end{array}$ & $\begin{array}{l}18.1(6.0- \\
30.2)\end{array}$ & $\begin{array}{l}17.8(6.7- \\
28.9)\end{array}$ & $\begin{array}{l}14.4(5.5- \\
23.2)\end{array}$ \\
\hline 14 & 3,377 & $\begin{array}{l}41.3(38.1- \\
44.4)\end{array}$ & $\begin{array}{l}28.9(26.6- \\
31.1)\end{array}$ & $\begin{array}{l}15.9(14.0- \\
17.7)\end{array}$ & $\begin{array}{l}14.0(11.2- \\
16.8)\end{array}$ \\
\hline 15 & 7,207 & $\begin{array}{l}44.9(42.1- \\
47.6)\end{array}$ & $\begin{array}{l}26.5(24.8- \\
28.2)\end{array}$ & $\begin{array}{l}16.7(15.4- \\
18.0)\end{array}$ & $\begin{array}{l}11.9(10.3- \\
13.6)\end{array}$ \\
\hline 16 & 7,416 & $\begin{array}{l}48.9(46.5- \\
51.4)\end{array}$ & $\begin{array}{l}25.6(24.0- \\
27.1)\end{array}$ & $\begin{array}{l}16.0(14.3- \\
17.8)\end{array}$ & $\begin{array}{l}9.5(8.3- \\
10.6)\end{array}$ \\
\hline 17 & 6,946 & $\begin{array}{l}52.9(50.4- \\
55.5)\end{array}$ & $\begin{array}{l}25.0(23.4- \\
26.7)\end{array}$ & $\begin{array}{l}14.1(12.9- \\
15.4)\end{array}$ & $7.9(6.7-9.1)$ \\
\hline$>=18$ & 3,773 & $\begin{array}{l}58.3(55.4- \\
61.3)\end{array}$ & $\begin{array}{l}23.5(21.6- \\
25.3)\end{array}$ & $\begin{array}{l}12.0(9.9- \\
14.1)\end{array}$ & $6.2(4.8-7.6)$ \\
\hline
\end{tabular}

\section{Race/Ethnicity}

\begin{tabular}{|c|c|c|c|c|c|}
\hline non-Hispanic white & 15,937 & $\begin{array}{l}45.5(42.0- \\
48.9)\end{array}$ & $\begin{array}{l}25.7(24.3- \\
27.1)\end{array}$ & $\begin{array}{l}17.0(15.4- \\
18.6)\end{array}$ & $\begin{array}{l}11.8(9.9- \\
13.7)\end{array}$ \\
\hline non-Hispanic black & 3,977 & $\begin{array}{l}52.4(50.2- \\
54.6)\end{array}$ & $\begin{array}{l}26.8(24.8- \\
28.8)\end{array}$ & $\begin{array}{l}14.2(12.7- \\
15.7)\end{array}$ & $6.6(5.5-7.6)$ \\
\hline Hispanic/Latino & 6,158 & $\begin{array}{l}55.6(53.6- \\
57.6)\end{array}$ & $\begin{array}{l}25.2(23.6- \\
26.7)\end{array}$ & $\begin{array}{l}11.8(10.7- \\
13.0)\end{array}$ & $7.4(6.4-8.4)$ \\
\hline Other ${ }^{a}$ & 2,712 & $\begin{array}{l}52.2(49.4- \\
54.9)\end{array}$ & $\begin{array}{l}26.0(24.1- \\
27.8)\end{array}$ & $\begin{array}{l}13.5(11.9- \\
15.1)\end{array}$ & $8.4(7.1-9.6)$ \\
\hline
\end{tabular}

\section{Weight status ${ }^{b}$}

Abbreviation: $\mathrm{Cl}$, confidence interval; MSA, muscle-strengthening activity; MVPA, moderate-to-vigorous intensity aerobic activity.

a Includes Native Hawaiian/Pacific Islander and non-Hispanic multiple race/ethnicities; bhealthy weight is defined as a body mass index (BMI) percentile from the 5 th to less than the 85th percentile, underweight is classified as a $\mathrm{BMI}<5$ th percentile, overweight is defined as a BMI percentile from the 85th to less than the 95th percentile, obese is defined as a BMI percentile equal or greater than the 95th percentile; ' Depressive symptoms is defined as reporting being sad for at least 2-weeks within the last year. 


\begin{tabular}{|c|c|c|c|c|c|}
\hline \multirow[t]{2}{*}{ Characteristic } & \multirow[t]{2}{*}{ Total } & \multicolumn{4}{|c|}{ Female sport participation, \% (95\% Cl) } \\
\hline & & 0 teams & 1 team & 2 teams & $\begin{array}{l}3 \text { or more } \\
\text { teams }\end{array}$ \\
\hline Healthy weight & 20,184 & $\begin{array}{l}45.7(43.3- \\
48.3)\end{array}$ & $\begin{array}{l}26.5(25.3- \\
27.7)\end{array}$ & $\begin{array}{l}16.5(15.4- \\
17.6)\end{array}$ & $\begin{array}{l}11.2(9.9- \\
12.6)\end{array}$ \\
\hline Under weight & 675 & $\begin{array}{l}59.1(53.0- \\
65.2)\end{array}$ & $\begin{array}{l}22.6(18.2- \\
27.1)\end{array}$ & $\begin{array}{l}11.6(71.6- \\
16.1)\end{array}$ & $6.6(4.1-9.2)$ \\
\hline Overweight & 4,681 & $\begin{array}{l}52.9(50.5- \\
55.4)\end{array}$ & $\begin{array}{l}25.1(23.4- \\
26.8)\end{array}$ & $\begin{array}{l}14.3(12.7- \\
16.0)\end{array}$ & $7.7(6.3-9.0)$ \\
\hline Obese & 3,244 & $\begin{array}{l}63.5(60.7- \\
66.4)\end{array}$ & $\begin{array}{l}22.7(20.5- \\
24.9)\end{array}$ & $\begin{array}{l}9.0(7.6- \\
10.4)\end{array}$ & $4.7(3.5-6.0)$ \\
\hline \multicolumn{6}{|l|}{ Screen use } \\
\hline$<2$ hours & 4,394 & $\begin{array}{l}46.1(41.3- \\
51.0)\end{array}$ & $\begin{array}{l}25.8(23.3- \\
28.2)\end{array}$ & $\begin{array}{l}16.5(14.6- \\
18.3)^{-}\end{array}$ & $\begin{array}{l}11.6(9.0- \\
14.3)\end{array}$ \\
\hline$>2$ hours & 24,390 & $\begin{array}{l}49.8(47.9- \\
51.7)\end{array}$ & $\begin{array}{l}25.8(24.8- \\
26.7)\end{array}$ & $\begin{array}{l}15.0(13.9- \\
16.0)\end{array}$ & $\begin{array}{l}9.5(8.5- \\
10.5)\end{array}$ \\
\hline \multicolumn{6}{|c|}{ Asthma diagnosis } \\
\hline No & 22,254 & $\begin{array}{l}50.0(47.8- \\
52.3)\end{array}$ & $\begin{array}{l}25.8(24.6- \\
27.0)\end{array}$ & $\begin{array}{l}14.7(13.7- \\
15.8)\end{array}$ & $\begin{array}{l}9.4(8.2- \\
10.7)\end{array}$ \\
\hline Yes & 6,530 & $\begin{array}{l}46.4(43.9- \\
48.8)\end{array}$ & $\begin{array}{l}25.6(24.1- \\
27.2)\end{array}$ & $\begin{array}{l}16.9(15.4- \\
18.4)\end{array}$ & $\begin{array}{l}11.1(9.7- \\
12.5)\end{array}$ \\
\hline \multicolumn{6}{|l|}{$\begin{array}{l}\text { Depressive } \\
\text { symptoms }^{c}\end{array}$} \\
\hline No & 11,224 & $\begin{array}{l}54.7(53.0- \\
56.4)\end{array}$ & $\begin{array}{l}24.6(23.3- \\
25.8)\end{array}$ & $\begin{array}{l}13.2(12.1- \\
14.3)\end{array}$ & $7.5(6.7-8.3)$ \\
\hline Yes & 17,560 & $\begin{array}{l}45.7(42.9- \\
48.6)\end{array}$ & $\begin{array}{l}26.5(25.2- \\
27.8)\end{array}$ & $\begin{array}{l}16.5(15.2- \\
17.7)\end{array}$ & $\begin{array}{l}11.3(9.6- \\
13.0)\end{array}$ \\
\hline \multicolumn{6}{|c|}{$\begin{array}{l}\text { Abbreviation: } \mathrm{Cl} \text {, confidence interval; MSA, muscle-strengthening activity; MVPA, moderate-to-vigorou } \\
\text { intensity aerobic activity. }\end{array}$} \\
\hline \multicolumn{6}{|c|}{$\begin{array}{l}\text { a Includes Native Hawaiian/Pacific Islander and non-Hispanic multiple race/ethnicities; }{ }^{\text {b }} \text { healthy } \\
\text { weight is defined as a body mass index (BMI) percentile from the } 5 \text { th to less than the } 85 \text { th percentile, } \\
\text { underweight is classified as a BMI <5th percentile, overweight is defined as a BMI percentile from the } \\
\text { 85th to less than the 95th percentile, obese is defined as a BMI percentile equal or greater than the } \\
\text { 95th percentile; }{ }^{\circ} \text { Depressive symptoms is defined as reporting being sad for at least } 2 \text {-weeks within } \\
\text { the last year. }\end{array}$} \\
\hline
\end{tabular}


Table 2

Weighted prevalence of sports-participation among male high school students residing in the United States by relevant characteristics, National Youth Risk Behavior Survey, 2011-2017

Characteristic

Total Male sport participation, \% (95\% Cl)

0 teams

29,260
38.6 (37.340.0)
1 team

$25.6(24.8-$
2 teams

$19.1(18.4-$ 19.8)

\section{3 or more} teams

$16.6(15.4-$ 17.9)

Age, years

\begin{tabular}{|c|c|c|c|c|c|}
\hline$<=13$ & 114 & $\begin{array}{l}45.9(32.6- \\
59.2)\end{array}$ & $\begin{array}{l}15.9(69.0- \\
24.9)\end{array}$ & $\begin{array}{l}17.9(7.4- \\
28.4)\end{array}$ & $\begin{array}{l}20.3(10.3- \\
30.2)\end{array}$ \\
\hline 14 & 2,926 & $\begin{array}{l}37.0(34.4-39- \\
6)\end{array}$ & $\begin{array}{l}26.3(24.0- \\
28.4)\end{array}$ & $\begin{array}{l}18.4(16.7- \\
20.3)\end{array}$ & $\begin{array}{l}18.3(16.1- \\
20.5)\end{array}$ \\
\hline 15 & 7,290 & $\begin{array}{l}34.7(32.3- \\
37.1)\end{array}$ & $\begin{array}{l}26.3(24.7- \\
28.0)\end{array}$ & $\begin{array}{l}19.9(18.4- \\
21.3)\end{array}$ & $\begin{array}{l}19.1(16.7- \\
21.6)\end{array}$ \\
\hline 16 & 7,369 & $\begin{array}{l}38.1(36.3- \\
40.0)\end{array}$ & $\begin{array}{l}25.2(23.9- \\
26.5)\end{array}$ & $\begin{array}{l}20.4(19.0- \\
22.0)\end{array}$ & $\begin{array}{l}16.2(14.7- \\
17.7)\end{array}$ \\
\hline 17 & 7,038 & $\begin{array}{l}40.7(38.7- \\
42.8)\end{array}$ & $\begin{array}{l}25.2(23.8- \\
26.7)\end{array}$ & $\begin{array}{l}18.5(17.2- \\
19.8)\end{array}$ & $\begin{array}{l}15.5(13.9- \\
17.0)\end{array}$ \\
\hline$>=18$ & 4,523 & $\begin{array}{l}43.5(41.0- \\
46.0)\end{array}$ & $\begin{array}{l}25.7(23.8- \\
27.6)\end{array}$ & $\begin{array}{l}16.8(15.1- \\
18.6)\end{array}$ & $\begin{array}{l}13.9(12.2- \\
15.6)\end{array}$ \\
\hline
\end{tabular}

Race/Ethnicity

\begin{tabular}{|c|c|c|c|c|c|}
\hline non-Hispanic white & 16,066 & $\begin{array}{l}38.0(36.2- \\
39.9)\end{array}$ & $\begin{array}{l}25.0(23.8- \\
26.3)\end{array}$ & $\begin{array}{l}18.7(17.8- \\
19.6)\end{array}$ & $\begin{array}{l}18.2(16.4- \\
20.0)\end{array}$ \\
\hline non-Hispanic black & 4,067 & $\begin{array}{l}34.3(32.2- \\
36.4)\end{array}$ & $\begin{array}{l}26.8(25.0- \\
28.6)\end{array}$ & $\begin{array}{l}22.0(20.2- \\
23.9)\end{array}$ & $\begin{array}{l}16.9(15.2- \\
18.7)\end{array}$ \\
\hline Hispanic/Latino & 6,358 & $\begin{array}{l}41.8(39.5- \\
44.0)\end{array}$ & $\begin{array}{l}26.7(25.3- \\
28.1)\end{array}$ & $\begin{array}{l}18.8(17.4- \\
20.2)\end{array}$ & $\begin{array}{l}12.7(11.7- \\
13.8)\end{array}$ \\
\hline Other ${ }^{a}$ & 2,769 & $\begin{array}{l}41.5(38.8- \\
44.2)\end{array}$ & $\begin{array}{l}25.0(22.8- \\
27.3)\end{array}$ & $\begin{array}{l}17.7(15.7- \\
19.7)\end{array}$ & $\begin{array}{l}15.8(13.6- \\
18.0)\end{array}$ \\
\hline
\end{tabular}

Weight status ${ }^{\text {b }}$

Abbreviation: $\mathrm{Cl}$, confidence interval; MSA, muscle-strengthening activity; MVPA, moderate-to-vigorous intensity aerobic activity.

a Includes Native Hawaiian/Pacific Islander and non-Hispanic multiple race/ethnicities; ${ }^{b}$ healthy weight is defined as a body mass index (BMI) percentile from the 5 th to less than the 85th percentile, underweight is classified as a $\mathrm{BMI}<5$ th percentile, overweight is defined as a BMI percentile from the 85th to less than the 95th percentile, obese is defined as a BMI percentile equal or greater than the 95th percentile; ' Depressive symptoms is defined as reporting being sad for at least 2-weeks within the last year. 


\begin{tabular}{|c|c|c|c|c|c|}
\hline \multirow[t]{2}{*}{ Characteristic } & \multirow[t]{2}{*}{ Total } & \multicolumn{4}{|c|}{ Male sport participation, \% (95\% Cl) } \\
\hline & & 0 teams & 1 team & 2 teams & $\begin{array}{l}3 \text { or more } \\
\text { teams }\end{array}$ \\
\hline Healthy weight & 18,795 & $\begin{array}{l}36.5(35.1- \\
38.0)\end{array}$ & $\begin{array}{l}25.8(24.8- \\
26.9)\end{array}$ & $\begin{array}{l}19.6(18.8- \\
20.4)\end{array}$ & $\begin{array}{l}18.1(16.6- \\
19.6)\end{array}$ \\
\hline Under weight & 1,014 & $\begin{array}{l}55.6(50.8- \\
60.5)\end{array}$ & $\begin{array}{l}21.4(18.0- \\
24.8)\end{array}$ & $\begin{array}{l}13.3(9.5- \\
17.0)\end{array}$ & $9.7(6.6-12.7)$ \\
\hline Overweight & 4,555 & $\begin{array}{l}37.1(34.9- \\
39.2)\end{array}$ & $\begin{array}{l}26.8(24.7- \\
28.8)\end{array}$ & $\begin{array}{l}20.0(18.3- \\
21.6)\end{array}$ & $\begin{array}{l}16.2(14.4- \\
18.0)\end{array}$ \\
\hline Obese & 4,896 & $\begin{array}{l}44.9(42.7- \\
47.0)\end{array}$ & $\begin{array}{l}24.8(22.9- \\
26.5)\end{array}$ & $\begin{array}{l}17.6(16.1- \\
19.1)\end{array}$ & $\begin{array}{l}12.9(11.2- \\
14.5)\end{array}$ \\
\hline \multicolumn{6}{|l|}{ Screen use } \\
\hline$<2$ hours & 3,828 & $\begin{array}{l}35.0(32.2- \\
37.8)\end{array}$ & $\begin{array}{l}25.9(23.8- \\
28.1)\end{array}$ & $\begin{array}{l}17.6(15.7- \\
19.4)\end{array}$ & $\begin{array}{l}21.5(18.8- \\
24.2)\end{array}$ \\
\hline$>2$ hours & 25,432 & $\begin{array}{l}39.2(37.6- \\
40.6)\end{array}$ & $\begin{array}{l}25.6(24.7- \\
26.5)\end{array}$ & $\begin{array}{l}19.3(18.6- \\
20.0)\end{array}$ & $\begin{array}{l}15.9(14.7- \\
17.1)^{(1)}\end{array}$ \\
\hline \multicolumn{6}{|c|}{ Asthma diagnosis } \\
\hline No & 22,715 & $\begin{array}{l}38.7(37.4- \\
40.1)\end{array}$ & $\begin{array}{l}25.9(25.0- \\
26.9)\end{array}$ & $\begin{array}{l}18.8(18.1- \\
19.6)\end{array}$ & $\begin{array}{l}16.4(15.2- \\
17.7)\end{array}$ \\
\hline Yes & 6,545 & $\begin{array}{l}38.3(36.1- \\
40.5)\end{array}$ & $\begin{array}{l}24.6(22.9- \\
26.2)\end{array}$ & $\begin{array}{l}20.0(18.5- \\
21.4)\end{array}$ & $\begin{array}{l}17.1(15.3- \\
18.9)^{(1)}\end{array}$ \\
\hline \multicolumn{6}{|l|}{$\begin{array}{l}\text { Depressive } \\
\text { symptoms }^{c}\end{array}$} \\
\hline No & 6,130 & $\begin{array}{l}45.7(43.6- \\
47.8)\end{array}$ & $\begin{array}{l}24.4(22.8- \\
26.1)\end{array}$ & $\begin{array}{l}16.1(14.8- \\
17.4)\end{array}$ & $\begin{array}{l}13.8(12.4- \\
15.2)\end{array}$ \\
\hline Yes & 23,130 & $\begin{array}{l}36.8(35.4- \\
38.2)\end{array}$ & $\begin{array}{l}26.0(25.1- \\
26.8)\end{array}$ & $\begin{array}{l}19.9(19.1- \\
20.6)\end{array}$ & $\begin{array}{l}17.3(16.0- \\
18.7)\end{array}$ \\
\hline \multicolumn{6}{|c|}{$\begin{array}{l}\text { Abbreviation: } \mathrm{Cl} \text {, confidence interval; MSA, muscle-strengthening activity; MVPA, moderate-to-vigorous } \\
\text { intensity aerobic activity. }\end{array}$} \\
\hline \multicolumn{6}{|c|}{$\begin{array}{l}\text { a Includes Native Hawaiian/Pacific Islander and non-Hispanic multiple race/ethnicities; bhealthy } \\
\text { weight is defined as a body mass index (BMI) percentile from the } 5 \text { th to less than the 85th percentile, } \\
\text { underweight is classified as a BMI<5th percentile, overweight is defined as a BMI percentile from the } \\
\text { 85th to less than the } 95 \text { th percentile, obese is defined as a BMI percentile equal or greater than the } \\
\text { 95th percentile; 'Depressive symptoms is defined as reporting being sad for at least 2-weeks within } \\
\text { the last year. }\end{array}$} \\
\hline
\end{tabular}

Among those who did not report participating in any sport, $2.5 \%(95 \% \mathrm{Cl}=2.2-2.9 \%)$ of females and $6.3 \%$ (95\% $\mathrm{Cl}=5.6 \%-7.0 \%)$ of males met the guidelines for sleep and physical activity (aerobic and musclestrengthening) (Table 3.). Meanwhile, $12.2 \%(95 \% \mathrm{Cl}=10.4 \%-14.0 \%)$ of females and $25.8 \%(95 \% \mathrm{Cl}=$ 
23.7\%-27.9\%) of males who participated in 3 or more sports met the guidelines for sleep and physical activity (aerobic and muscle-strengthening). For males, those who reported playing sports had higher proportions of meeting the sleep and physical activity guidelines, both independently and in combination, compared to those who did not play sports. For females, results were similar to those for males for estimates of meeting the physical activity guidelines, but not for meeting the sleep guidelines. Among those who did not participate in sports, $29.5 \%(95 \% \mathrm{Cl}=28.3-30.6)$ met the sleep recommendations, while estimates of those participating in 1 sport (30.1\% [95\% Cl $=28.5 \%-31.7 \%]), 2$ sports $(31.0 \%$ [95\% Cl $=28.5 \%-31.7 \%])$, and 3 sports or more $(28.4 \%[95 \% \mathrm{Cl}=28.4 \%-34.7 \%)$ were similar. 
Table 3

Weighted prevalence of meeting sleep and physical activity recommendations among American high school students participating in sports by sex, National Youth Risk Behavior Survey, 2011-2017.

\section{Students meeting recommendations, \% (95\% Cl)}

0 teams

1 team

2 teams

3 or more teams

\section{Females}

Sleep

$29.5(28.3-$

30.6)

Physical activity

MVPA

$9.9(9.1-10.7)$

$17.4(16.1-$

18.7)

MSA

MVPA + MSA

$51.4(49.6-$

53.1)

$7.2(6.5-7.9)$

$71.5(69.9-$

73.1)

14.9 (13.7-

16.1)
31.0 (28.7-

33.3)
$31.5(28.4-$

34.7)
$28.7(26.2-$

31.2)

$82.7(81.0-84.5)$

25.9 (23.3-

28.6)
$42.7(40.0-45.5)$

89.0 (87.1-

90.8)

39.6 (36.9-

42.3)

Physical activity +

Sleep

MVPA + Sleep

$3.5(3.0-3.9)$

$5.9(5.1-6.7)$

$9.1(7.8-10.3)$

$13.4(11.4-$

15.3)

MSA + Sleep

$12.4(11.6-$

$19.3(17.9-$

20.6)

$23.6(21.5-$

13.3)

$5.2(4.3-5.8)$

25.7)

25.7 (22.8-

28.6)

MVPA + MSA + Sleep

$2.5(2.2-2.9)$

$8.2(7.0-9.3)$

12.2 (10.4-

14.0)

Males

Sleep

$30.2(28.9-$

31.6)

35.7 (34.1-

37.4)

38.9 (36.7-

41.2)

$41.4(39.0-43.7)$

Physical activity

MVPA

21.0 (19.8-

22.2)

35.7 (34.2-

37.2)

$45.9(43.9-$

48.0)

63.5 (61.3-

65.6)

MSA

$64.0(62.4-$

65.6)

83.6 (82.3-

84.9)

90.7 (89.6-

91.9)

92.4 (91.1-

93.7)

MVPA + MSA

17.5 (16.5-

33.1 (31.6-

44.1 (42.1-

60.3 (58.2-

18.6)

34.6)

46.1)

62.5)

Physical activity +

Sleep

MVPA + Sleep

$7.6(6.9-8.4)$

$14.3(13.2-$

15.4)

$20.3(18.5-$

27.5 (25.4-

29.6) 


\begin{tabular}{|lllll|}
\hline \multicolumn{5}{|l}{ Students meeting recommendations, \% $(\mathbf{9 5 \%} \mathbf{~ C l})$} \\
\cline { 2 - 5 } & $\mathbf{0}$ teams & $\mathbf{1}$ team & $\mathbf{2}$ teams & 3 or more teams \\
\hline MSA + Sleep & $17.9(16.8-$ & $28.8(27.3-$ & $34.4(32.1-$ & $37.1(34.9-$ \\
& $19.1)$ & $30.4)$ & $36.6)$ & $39.3)$ \\
MVPA + MSA + Sleep & $6.3(5.6-7.0)$ & $13.2(12.1-$ & $19.6(17.9-$ & $25.8(23.7-$ \\
& & $14.3)$ & $21.4)$ & $27.9)$ \\
\hline
\end{tabular}

The relative odds of meeting the sleep and physical activity recommendations among American high school students participating in sports are presented by sex in Figs. 1 (female) and 2 (male). Results indicate that among both females and males, sports participation is associated with significantly higher odds of meeting the recommendations for time spent sleeping and engaging in physical activity concurrently. Specifically, among females, those who play 1 sport are $1.95(95 \% \mathrm{Cl}=1.59-2.40)$ times more likely to achieve the recommendations for sleeping and physical activity (aerobic and musclestrengthening) compared to those playing no sports, after controlling for age, race/ethnicity, weight status, screen-use, and comorbidities (asthma, and depressive symptoms). Measures of effect were consistently larger for the greater number of sports played, indicating a possible dose-response relation. Meeting the recommended time sleeping was not associated with sports participation among females, however. Results were similar for males, except sport participants were significantly more likely to meet sleep guidelines compared to those that play no sports. Additionally, the measures of effect were generally greater for males than females. Interestingly, the strongest associations across all the recommendations for females and males were for muscle-strengthening physical activity both independently, and in combination with aerobic physical activity. For females, those who participated in 3 or more sports, were $7.82(95 \% \mathrm{Cl}=6.68-8.36)$ times more likely to meet the recommendations for physical activity (aerobic and muscle-strengthening) compared to those who do not play sports, after controlling for the covariates of interest (results not shown in Fig. 1). For males, those who participated in 3 or more sports, were $6.94(95 \% \mathrm{Cl}=6.20-7.76)$ times more likely to meet the recommendations for physical activity (aerobic and muscle-strengthening) compared to those who did not play sports, after controlling for the covariates of interest (results not shown in Fig. 2).

Figure 1. Relative odds of meeting sleep and physical activity recommendations among female American high school students participating in sports, National Youth Risk Behavior Survey, 2011-2017.

Figure 2. Relative odds of meeting sleep and physical activity recommendations among male American high school students participating in sports, National Youth Risk Behavior Survey, 2011-2017.

\section{Discussion}

The purpose of this paper was to determine whether participation in high school sports was associated with concurrently and independently achieving the recommended levels of sleep and physical activity among a nationally representative sample of American adolescents. Significant associations were found 
between sports participation and meeting sleep and physical activity (aerobic and muscle-strengthening) guidelines. Furthermore, a dose-response relation was seen for the number of sports played in the past year and the relative odds of meeting all three guidelines, with a strong association between sports participation and meeting physical activity guidelines.

The significant associations for sports participation and meeting guidelines align with those from a study by Mäkelä et al, who compared physical activity, sleep time, and screen time between Finnish sports club participants and non-participants (ages 14-16).(15) They found that sports participants (versus nonparticipants) had significantly higher odds of meeting aerobic physical activity guidelines, sleeping $\geq 9$ hours on the weekends, and having $<2$ hours daily screen time. Sports provide an opportunity for youth to be physically active, so it follows that students who participate in sports have been found to be more likely to meet aerobic physical activity guidelines than non-participants.(16) Further, it has been found that adolescents with higher (versus lower) physical activity levels are more likely to have better sleep quality and quantity,(6) including among athletes versus non-athletes,(17-20) which also aligns with the presented findings. The strong association between sports participation and meeting musclestrengthening guidelines may be due to more opportunities for athletes (versus non-athletes) to engage in muscle-strengthening activities within both training and game sessions. Muscle-strengthening activities (e.g., push-ups, sit-ups, weight-lifting) are often incorporated into sports-related training, but are less frequently seen in the other common physical activities that youth engage in outside of sports (e.g., walking, cycling, running).(21)

The association between sports participation and meeting sleep and physical activity guidelines had not previously been examined with consideration for the number of sports played, as done in the present study. The dose-response relationship that emerged for all guidelines among males and all but sleep among females is a valuable contribution to the literature. These findings suggest there are more benefits with playing multiple sports during the year, versus single-sport or non-participation, although additional research is needed to examine whether this dose-response relation holds when adolescents play multiple sports concurrently, versus at different times throughout the year. These findings also suggest that the binary categorization of sports participation (yes/no) often seen in research may not adequately capture this construct, and future research should take this into consideration.

The findings for racial/ethnic and sex differences, with a higher prevalence of sport participation among non-Hispanic whites (versus all other racial/ethnic groups) and among males (versus females), align with those often seen for physical activity levels and sports participation among these sociodemographic groups. $(15,22)$ However, future research should further examine why such disparities persist within the associations of sports participation and meeting guidelines. For example, although a significant association between sports participation and meeting sleep guidelines was found among males, this association was not significant for females. Increasing the understanding of these sex and racial/ethnic disparities, as well as reducing barriers to sports participation, could help efforts to equitably increase the prevalence of youth sports participation and the associated health benefits. 


\section{Strengths and Limitations.}

The strengths of this study include the inclusion of a large, population-based sample, which provides external study validity, as well as the use of multiple imputation to address potential bias due to missing data. This study also addresses numerous gaps in the literature. Few studies have examined the association of sports participation with sleep and physical activity, both independently and concurrently. Furthermore, the number of sports played was included in the measure of sports participation, allowing for a greater understanding of its relationship with meeting guidelines - namely, the dose-response relation that emerged. Such findings highlight sports participation as a potential method for addressing the low prevalence of youth meeting sleep and physical activity guidelines. For example, although sleep interventions have been implemented among elite athletes,(23) few school-based policies aiming to improve sleep among students exist. However, schools could consider sports participation as a method for increasing sleep quantity, as well as reducing screen time, among this population.

The limitations of this study must also be noted. First, the YRBS is a cross-sectional survey, which limits the ability to make causal inferences between the exposures and outcomes under study. Second, the selfreported items in YRBS are prone to recall bias and potential social desirability bias. Third, the YRBS does not contain a measure of socioeconomic status (SES) so it was not possible to examine potential differences by SES. Fourth, the time-frame recall was different for physical activity (past 7 days), sleep (average for school night), and sports participation (past 12 months), and it was not possible to determine if any of the sports were played concurrently. However, although some respondents classified as sports participants may not have been actively engaged in sports at the time of the survey, significant associations were found. This could suggest that sports participants are engaging in healthy sleep and physical activity behaviors during non-sport periods as well, although additional research is needed to explore this hypothesis. Finally, neither the sport type nor competitive level (e.g., recreational, club, etc.) were included within the YRBS, and findings may differ across these groups due to the variations in intensity.(24) This may have also played a role in the disparities seen for sex, considering the distribution of males and females across sports played and level of participation.(22) Future research could expand on the few studies examining youth demographics by type of sport, with additional consideration for its role in the association of sports participation and meeting guidelines.

The reported findings demonstrate significant associations of sports participation and achieving the recommended levels of sleep and physical activity (both independently and concurrently). Efforts to increase the prevalence of youth meeting guidelines should consider promoting sports participation while reducing barriers to participate, particularly among females and racial/ethnic minorities. Practitioners can respond to concerns from parents and youth about potential risk of injury by emphasizing the benefits of sports participation, including those related to screen time and mental and physical health.

\section{Declarations}

Ethics approval and consent to participate. 
This study protocol was reviewed and received exempt status by The University of Texas Health Science Center at Houston (UTHealth) Institutional Review Board.

\section{Consent for publication.}

Not applicable.

\section{Availability of data and materials.}

The datasets generated and/or analysed during the current study are available in the Centers for Disease Control and Prevention repository, https://www.cdc.gov/healthyyouth/data/yrbs/data.htm.

\section{Competing interests.}

The authors declare that they have no competing interests

\section{Funding.}

Dr Walker was supported by a research career development award for (K12HD052023): Building Interdisciplinary Research Career in Women's Health Program-BIRCHW; Berenson, PI) from the Eunice Kennedy Shriver National Institute of Child Health and Human Development (NICHD) at the National Institutes of Health. The content is solely the responsibility of the authors and does not necessarily represent the official views of the National Institutes of Health.

\section{Authors' contributions.}

GK was responsible for the concept and design of the study; acquisition, analysis, and interpretation of the data; drafting and revision of the manuscript. TW contributed to the concept and design of the study; drafting and revision of the manuscript. AJ and SB contributed to the drafting and revision of the manuscript; JP supervised the project and contributed to the drafting and revision of the manuscript.

\section{References}

1. Kuo BD, Goodman E. The Power and Promise of Youth's Voices. Childhood Obesity. 2011;7(1):16.

2. Pesonen A-K, Sjöstén NM, Matthews KA, Heinonen K, Martikainen S, Kajantie E, et al. Temporal associations between daytime physical activity and sleep in children. PloS one. 2011;6(8):e222958-e.

3. Stone MR, Stevens D, Faulkner GEJ. Maintaining recommended sleep throughout the week is associated with increased physical activity in children. Prev Med. 2013;56(2):112-7.

4. Roman-Viñas B, Chaput J-P, Katzmarzyk PT, Fogelholm M, Lambert EV, Maher C, et al. Proportion of children meeting recommendations for 24-hour movement guidelines and associations with adiposity in a 12-country study. International Journal of Behavioral Nutrition Physical Activity. 2016;13(1):123. 
5. Zhu X, Healy S, Haegele JA, Patterson F. Twenty-Four-Hour Movement Guidelines and Body Weight in Youth. J Pediatr. 2020;218:204-9.

6. Lang C, Kalak N, Brand S, Holsboer-Trachsler E, Pühse U, Gerber M. The relationship between physical activity and sleep from mid adolescence to early adulthood. A systematic review of methodological approaches and meta-analysis. Sleep Med Rev. 2016;28:32-45.

7. Dolezal BA, Neufeld EV, Boland DM, Martin JL, Cooper CB. Interrelationship between Sleep and Exercise: A Systematic Review. Advances in Preventive Medicine. 2017;2017:1364387.

8. Wang F, Boros S. The effect of physical activity on sleep quality: a systematic review. European Journal of Physiotherapy. 2019:1-8.

9. Baldursdottir B, Taehtinen RE, Sigfusdottir ID, Krettek A, Valdimarsdottir HB. Impact of a physical activity intervention on adolescents' subjective sleep quality: a pilot study. Global health promotion. 2017;24(4):14-22.

10. Harris A, Gundersen H, Andreassen PM, Thun E, Bjorvatn B, Pallesen S. A Comparative Study of Sleep and Mood Between Young Elite Athletes and Age-Matched Controls. J Phys Act Health. 2017;14(6):465-73.

11. Messing S, Rütten A, Abu-Omar K, Ungerer-Röhrich U, Goodwin L, Burlacu I, et al. How Can Physical Activity Be Promoted Among Children and Adolescents? A Systematic Review of Reviews Across Settings. Frontiers in public health. 2019;7:55-.

12. Kann LMT, Harris WA, Shanklin SL, Flint KH, Quee B, Lowry R, Chyen D, Whittle L, Thorton J, Lim C, Bradford D, Yamakawa Y, Leon M, Brener N, Ethier KA. Youth Risk Behavior Surveillance - United States, 2017. Morb Mortal Wkly Rep. 2018;67(8):1-114.

13. Hirshkowitz M, Whiton K, Albert SM, Alessi C, Bruni O, DonCarlos L, et al. National Sleep Foundation's sleep time duration recommendations: methodology and results summary. Sleep health. 2015;1(1):40-3.

14. White IR, Royston P, Wood AM. Multiple imputation using chained equations: issues and guidance for practice. Statistics in medicine. 2011;30(4):377-99.

15. Mäkelä K, Kokko S, Kannas L, Villberg J, Vasankari T, Heinonen JO, et al. Physical activity, screen time and sleep among youth participating and non-participating in organized sports: The finnish health promoting sports club (FHPSC) study. Advances in Physical Education. 2016;6.

16. Mandic S, Bengoechea EG, Stevens E, de la Barra SL, Skidmore P. Getting kids active by participating in sport and doing it more often: focusing on what matters. International journal of behavioral nutrition physical activity. 2012;9(1):86.

17. Brand S, Beck J, Gerber M, Hatzinger M, Holsboer-Trachsler E. 'Football Is Good for Your Sleep' Favorable Sleep Patterns and Psychological Functioning of Adolescent Male Intense Football Players Compared to Controls. J Health Psychol. 2009;14(8):1144-55.

18. Brand S, Gerber M, Beck J, Hatzinger M, Pühse U, Holsboer-Trachsler E. High exercise levels are related to favorable sleep patterns and psychological functioning in adolescents: a comparison of athletes and controls. J Adolesc Health. 2010;46(2):133-41. 
19. Brand S, Beck J, Gerber M, Hatzinger M, Holsboer-Trachsler E. Evidence of favorable sleep-EEG patterns in adolescent male vigorous football players compared to controls. The world journal of biological psychiatry. 2010;11(2-2):465-75.

20. Weydahl A. Sleep-quality among girls with different involvement in competitive sports during fall in the arctic circle. Percept Mot Skills. 1991;73(3):883-92.

21. Fakhouri TH, Hughes JP, Burt VL, Song M, Fulton JE, Ogden CL. Physical Activity in US Youth Aged 12-15 Years, 2012. NCHS Data Brief. Number 141. Centers for Disease Control and Prevention. 2014.

22. Turner RW, Perrin EM, Coyne-Beasley T, Peterson CJ, Skinner AC. Reported Sports Participation, Race, Sex, Ethnicity, and Obesity in US Adolescents From NHANES Physical Activity (PAQ_D). Global pediatric health. 2015;2:2333794 × 15577944 .

23. Bonnar D, Bartel K, Kakoschke N, Lang C. Sleep Interventions Designed to Improve Athletic Performance and Recovery: A Systematic Review of Current Approaches. Sports medicine (Auckland, NZ). 2018;48(3):683-703.

24. Butte NF, Watson KB, Ridley K, Zakeri IF, McMurray RG, Pfeiffer KA, et al. A Youth Compendium of Physical Activities: Activity Codes and Metabolic Intensities. Med Sci Sports Exerc. 2018;50(2):24656.

\section{Figures}

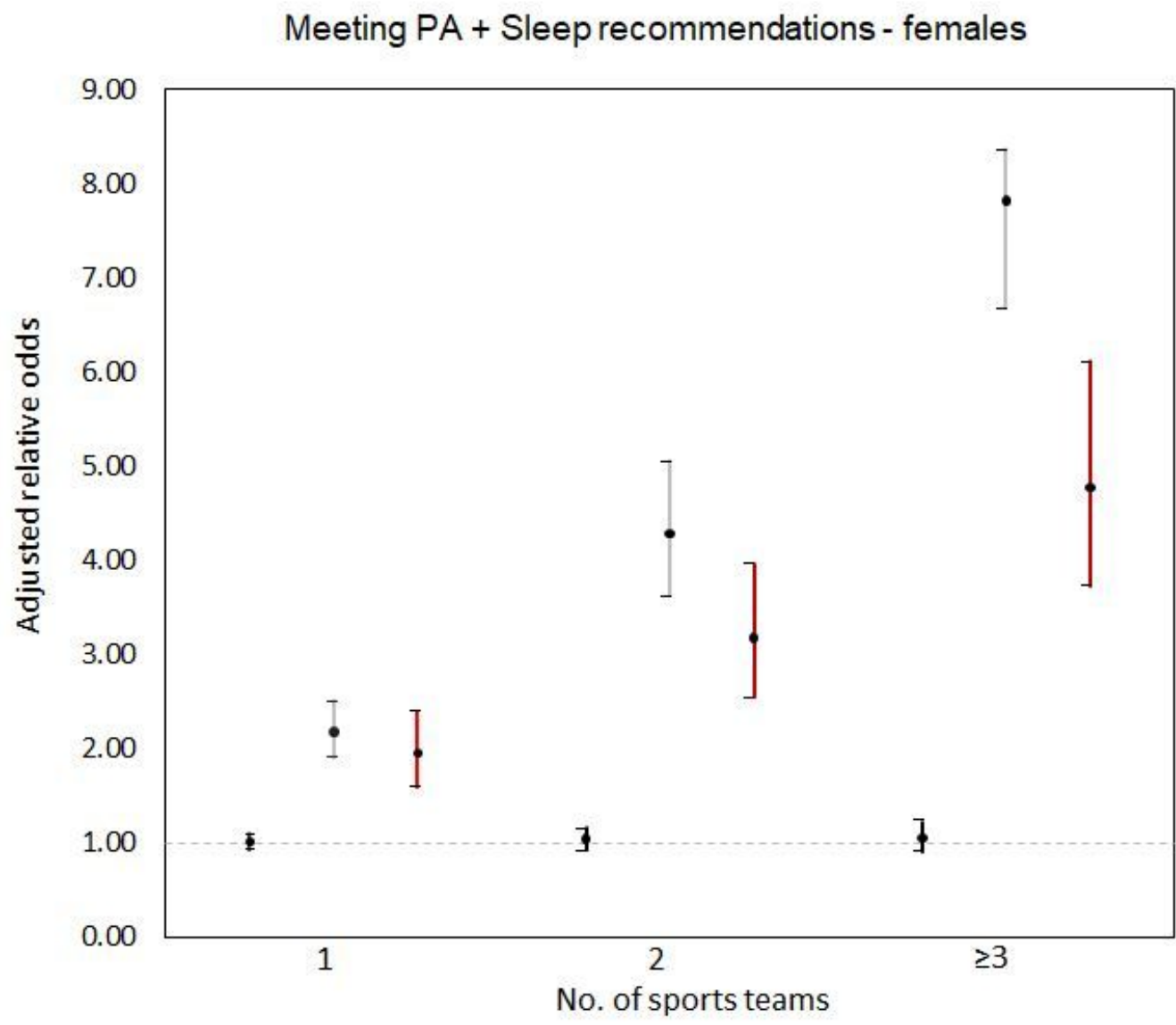

\section{Legend}

Sleep

Physical activity (MSA+MVPA)

Sleep + physical activity (MSA+MVPA) 
Relative odds of meeting sleep and physical activity recommendations among female American high school students participating in sports, National Youth Risk Behavior Survey, 2011-2017.

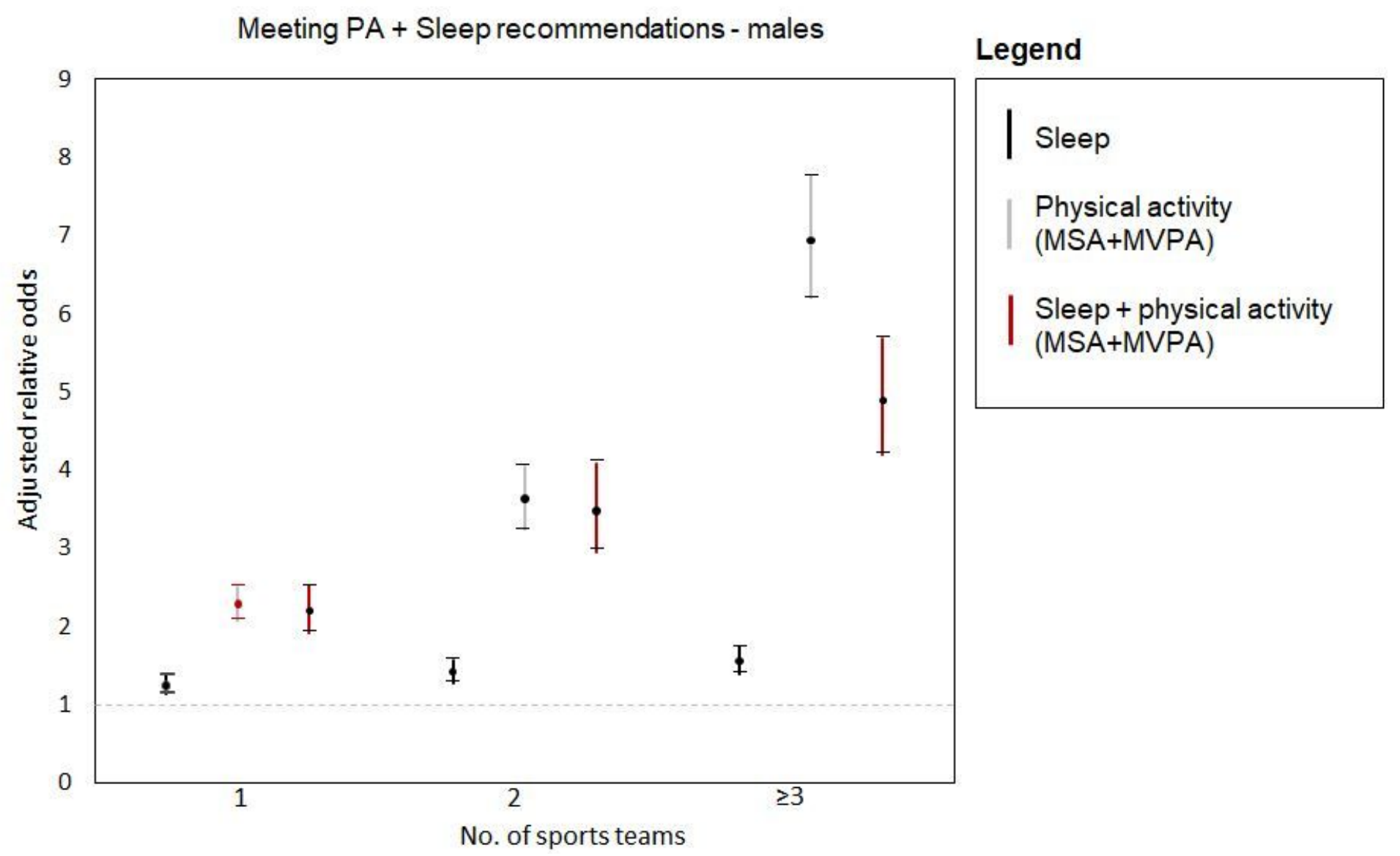

Figure 2

Relative odds of meeting sleep and physical activity recommendations among male American high school students participating in sports, National Youth Risk Behavior Survey, 2011-2017. 